\title{
The analysis and design of a 50MHz Colpitts low phase noise crystal oscillator.
}

\author{
Yan Wang*, Ying Cui, Shiyi Xu
}

School of Electrical Engineering and Information, Southwest Petroleum University, No.8 Xindu Avenue, Xindu District, Chengdu City, Sichuan Province, PR China

\begin{abstract}
Crystal oscillators have huge demand in medical filed especially in building medical devices. They are used in diagnostic equipment, monitoring devices, hearing aids, etc. Since they are widely used in medical devices, it is essential to maintain accurate requirements for the output phase noise of reference frequency source. At the same time it is important to find out new ways to reduce the phase noise which is the major problem in designing crystal oscillators. The importance of loaded quality factor $Q_{\mathrm{L}}$ is analyzed on the basis of Leeson model and the formula of $Q_{\mathrm{L}}$ is derived from the analysis of Colpitts oscillator circuit. According to the results, we can draw a conclusion that $Q_{\mathrm{L}}$ is explicitly related to circuit parameters. The phase noise of the $50 \mathrm{MHz}$ Colpitts crystal oscillator is simulated by the Agilent Advanced Design System (ADS). Based on the simulation results, a design of the prototype crystal oscillator is presented. The crystal resonator we use is AT-cut $3^{\text {rd }}$ overtone crystal resonator with $49 \mathrm{U}$ resistance welding package, its unloaded quality factor $Q_{0}$ is about $1.45 \times 10^{5}$. The measured results of phase noise are $-107 \mathrm{dBc} / \mathrm{Hz} @ 10 \mathrm{~Hz},-134 \mathrm{dBc} / \mathrm{Hz} @ 100 \mathrm{~Hz}$ and $-152 \mathrm{dBc} / \mathrm{Hz} @ 1 \mathrm{KHz}$. Experimental results show that it is feasible to design low phase noise crystal oscillators on the basis of improving $Q_{\mathrm{L}}$.
\end{abstract}

Keywords: Leeson model, Loaded quality factor, Phase noise, Colpitts crystal oscillator.

Accepted on August 30, 2017

\section{Introduction}

With the development of modern communication technology, the requirement of the high stable crystal oscillator has increased. Crystal oscillators have numerous applications and they are widely used in medical devices such as defibrillators, neuro-stimulators, hearing aids, pacemakers, etc. [1]. They are also used in ultrasound, MRI equipment, wireless telemetry and diagnostic imaging. Neurologists often use oscillators for treating neurological disorders such as Parkinson's Disease through deep brain stimulation (DBS) [2,3]. Functional pacemakers coordinating circadian rhythms are constructed by coupling multiple oscillators. Circadian oscillators constitute positive and negative elements which can form autoregulatory feedback loops. These loops are used to generate 24-hour timing circuits $[4,5]$. Patients with middle ear dysfunction use Bone Anchored Hearing Aids which contains bone oscillators [6]. In many systems such as the medical field, satellite communication and radar system, there are very strict accuracy requirements for the output phase noise of reference frequency source. Therefore, the study of ways to reduce the phase noise is the primary problem in the process of design crystal oscillators [7-9].

In practical design, the phase noise is closely related to $Q_{\mathrm{L}}$. By the analysis of Leeson formula derived from the phase noise model of Leeson, we can draw a conclusion that phase noise improves with the loaded quality factor $Q_{\mathrm{L}}$ increasing. The Leeson formula is as follows [10-13]:

$S_{\Delta \phi}\left(f_{m}\right)=\left[1+\frac{1}{f_{m}^{2}}\left(\frac{f_{0}}{2 Q_{L}}\right)^{2}\right] S_{\Delta \theta}\left(f_{m}\right) \rightarrow(1)$

Where $f_{\mathrm{m}}$ is offset frequency, $f_{0}$ is center frequency, $Q_{\mathrm{L}}$ is loaded quality factor, $S_{\Delta \phi}\left(f_{\mathrm{m}}\right)$ is the power spectral density of the output phase noise, $S_{\Delta \phi}\left(f_{\mathrm{m}}\right)$ is the spectral density of the oscillator input phase noise. So a high $Q_{\mathrm{L}}$ is beneficial to reducing phase noise.

In this paper, the formula of $Q_{\mathrm{L}}$ is derived by the analysis of Colpitts oscillator circuit and we can obtain the relation between $Q_{\mathrm{L}}$ and circuit parameters. An appropriate increase in the value of capacitance $C_{2}$ can improve $Q_{\mathrm{L}}$, thereby reduce the phase noise. According to the simulation results of $50 \mathrm{MHz}$ Colpitts crystal oscillator using Agilent Advanced Design System (ADS), a design of the prototype crystal oscillator is presented and the measurement results of phase noise are reduced by adjusting the value of $C_{2}$.

\section{The phase noise analysis of colpitts crystal oscillator}

From the viewpoint of oscillator power spectrum, for BJT oscillator, the $Q_{\mathrm{L}}$ factor of a conventional two-port network is defined as [14-16]: 
$Q_{L}=\frac{\omega}{2}\left|\frac{1}{z(\omega)} \times \frac{d z(\omega)}{d \omega}\right|_{\omega=\omega_{0}}=\frac{\omega}{2}\left|\frac{d}{d \omega} \ln \frac{z_{11}(\omega)}{z_{12}(\omega)}\right|_{\omega=\omega_{0}}$

Where $z_{11}(\omega)$ and $z_{12}(\omega)$ are terms of the circuit impedance matrix Z.

As an example, a modified $50 \mathrm{MHz}$ Colpitts low phase noise crystal oscillator is discussed [17]. The equivalent circuit is shown in Figure 1.

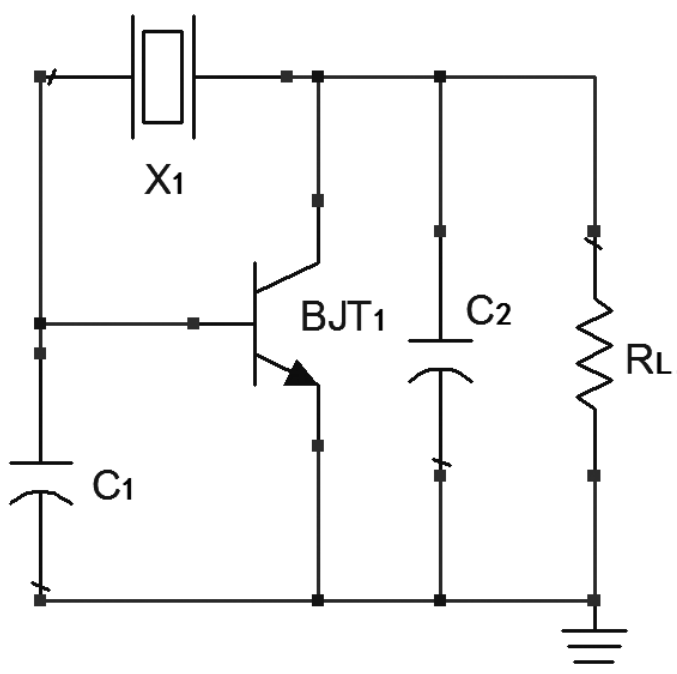

Figure 1. The equivalent circuit of Colpitts crystal oscillator.

In the above circuit, the crystal resonator $\mathrm{X} 1$ acts as an inductance L1. The equivalent circuit of passive network is shown in Figure 2.

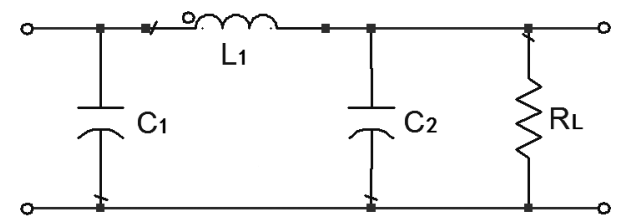

Figure 2. The equivalent circuit of passive network.

The impedance matrix $\mathrm{Z}$ of passive network in the circuit is expressed as follows:

$Z=\frac{1}{\left(1-\omega^{2} L_{1} C_{1}\right)\left(1+j \omega C_{2} R_{L}\right)+j \omega C_{1} R_{L}}$
$\left(\begin{array}{cc}j \omega L_{1}\left(1+j \omega C_{2} R_{L}\right)+R_{L} & R_{L} \\ R_{L} & R_{L}-\omega^{2} L_{1} C_{1} R_{L}\end{array}\right)$

According to (2) and (3), the result of $Q_{\mathrm{L}}$ is as follows:

$$
\begin{aligned}
& Q_{L}=\frac{\omega}{2}\left|\frac{d}{d \omega} \ln \frac{z_{11}(\omega)}{z_{12}(\omega)}\right|_{\omega=\omega_{0}} \\
& =\frac{\omega_{0}}{2} \sqrt{\frac{L_{1}^{2}\left(4 \omega_{0}^{2} C_{2}^{2} R_{L}^{2}+1\right)}{R_{L}^{2}+\omega_{0}^{2} L_{1}^{2}+\omega_{0}^{4} L_{1}^{2} C_{2}^{2} R_{L}^{2}-2 \omega_{0}^{2} L_{1} C_{2} R_{L}}} \rightarrow(4)
\end{aligned}
$$

From (4), we can draw a conclusion that $Q_{\mathrm{L}}$ would increase with $C_{2}$, that is to say, the larger $C_{2}$, the higher $Q_{\mathrm{L}}$.

\section{The simulation and design of colpitts crystal oscillator}

The $50 \mathrm{MHz}$ Colpitts crystal oscillator that its equivalent circuit is shown in Figure 2 is simulated by software ADS and the simulated phase noise curve is shown in Figure 3.

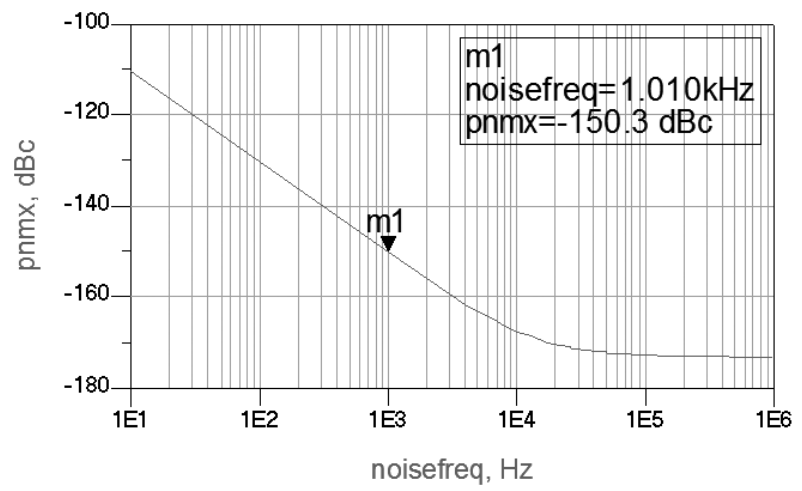

Figure 3. The simulated phase noise curve.

The value of $C_{2}$ is increased appropriately to improve the value of $Q_{\mathrm{L}}$ and reduce the phase noise. The simulated phase noise curve after adjusting $C_{2}$ is also presented in Figure 4 .

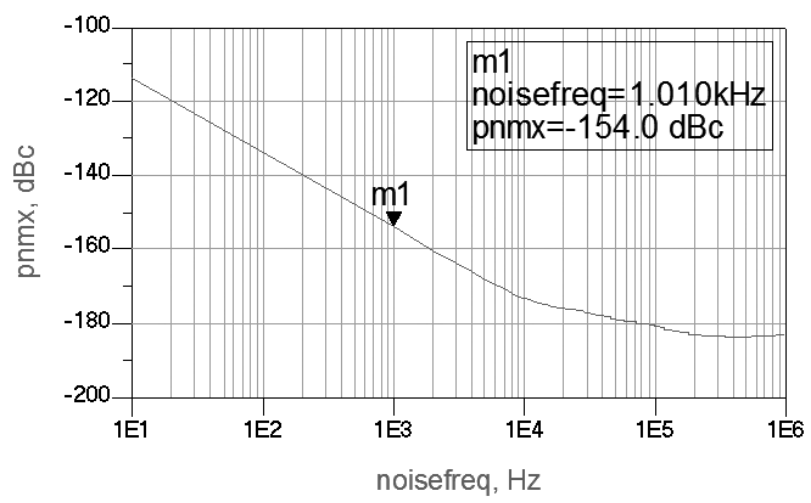

Figure 4. The simulated phase noise curve after adjusting $C 2$.

Table 1. The parameters of crystal resonator.

\begin{tabular}{ll}
\hline Parameter & Value \\
\hline $\mathrm{Lq}$ & $0.012 \mathrm{H}$ \\
\hline $\mathrm{rq}$ & $26 \Omega$ \\
\hline $\mathrm{Cq}$ & $8.4 \times 10^{-16 \mathrm{~F}}$ \\
\hline $\mathrm{C} 0$ & $2.2 \times 10^{-12 \mathrm{~F}}$
\end{tabular}

According to the simulation results by ADS, the phase noise is decreased after adjusting $C_{2}$. Meanwhile, we can draw the conclusion that an appropriate increase in the value of $C_{2}$ will help to reduce the phase noise of this circuit. However, if $C_{2}$ is too large, the oscillation condition of the amplitude cannot be met and it is difficult to produce oscillation. Therefore in the allowable range, $C_{2}$ should be made as high as possible to 
reduce the phase noise. Based on the conclusions above, a prototype $50 \mathrm{MHz}$ Colpitts crystal oscillator is designed. The AT-cut $3^{\text {rd }}$ overtone crystal resonator with $49 \mathrm{U}$ resistance welding package is used in the design and its quality is medium. Its parameters are as Table 1 and the value of unloaded quality factor $Q_{0}$ is about $1.45 \times 10^{5}$. The phase noise level of the $50 \mathrm{MHz}$ Colpitts crystal oscillator is measured by Agilent E5052B and it is shown in Figure 5.

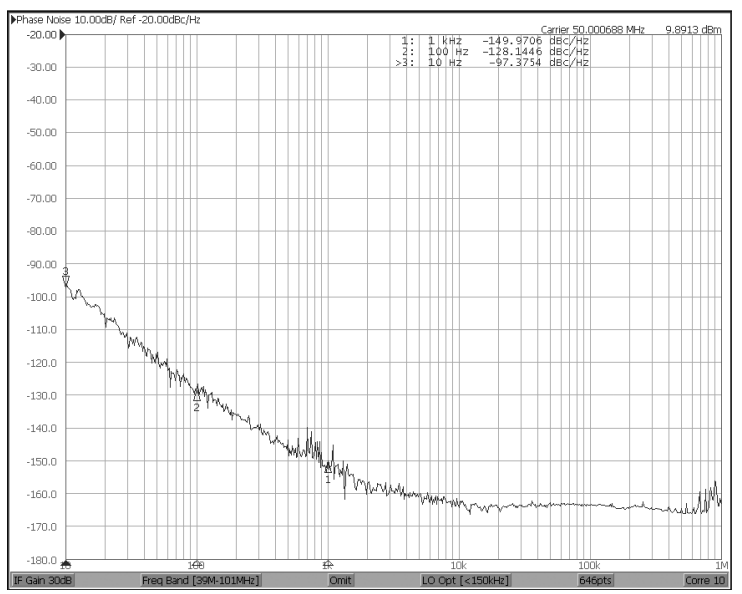

Figure 5. Measured phase noise curve of the $50 \mathrm{MHz}$ crystal oscillator

As in Figure 5, the measured results of phase noise are -97 $\mathrm{dBc} / \mathrm{Hz} @ 10 \mathrm{~Hz}, \quad-128 \mathrm{dBc} / \mathrm{Hz} @ 100 \mathrm{~Hz}$ and $-149 \mathrm{dBc} /$ $\mathrm{Hz} @ 1 \mathrm{KHz}$. Based on above analysis, the value of capacitance $\mathrm{C} 2$ is adjusted to reduce the phase noise further. The measured results of phase noise level after adjusting $C_{2}$ are $-107 \mathrm{dBc} /$ $\mathrm{Hz} @ 10 \mathrm{~Hz},-134 \mathrm{dBc} / \mathrm{Hz} @ 100 \mathrm{~Hz}$ and $-152 \mathrm{dBc} / \mathrm{Hz} @ 1 \mathrm{KHz}$ and it is shown in Figure 6.

According to Figure 6, the phase noise is improving by adjusting the value of capacitance $C_{2}$. Though the crystal resonator used in the design is medium, the measured results of phase noise are relatively well and the crystal resonator's performance is realized as far as possible by the design based on improving $Q_{\mathrm{L}}$.

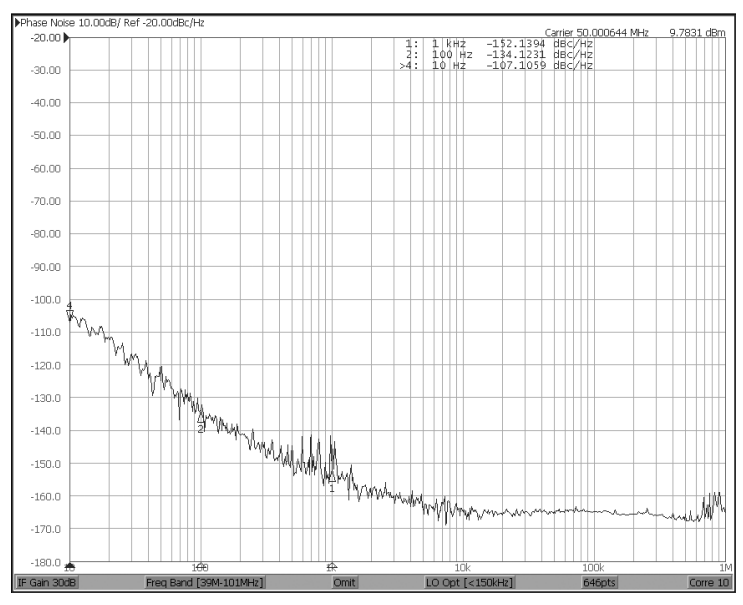

Figure 6. Measured phase noise curve after adjusting $C_{2}$.

\section{Conclusion}

In this paper, the $50 \mathrm{MHz}$ Colpitts crystal oscillator is designed on the basis of improving $Q_{\mathrm{L}}$. Based on the simulation results by $\mathrm{ADS}$, a design of the prototype $50 \mathrm{MHz}$ crystal oscillator is presented and the experiments are carried out. By adjusting the value of capacitance $C_{2}$, the phase noise level is further improved and the performance of the crystal resonator is realized as far as possible. So it is feasible to design low phase noise crystal oscillators on the basis of improving $Q_{\mathrm{L}}$.

\section{Acknowledgment}

This work is supported by application fundamental research fund of SiChuan Provincial Science and Technology Department (No. 2014JY0208) and scientific research starting project of SWPU (No. 2014QHZ026).

\section{References}

1. https://www.elprocus.com/crystal-oscillator-circuit-andworking/

2. Wilson D, Moehlis J. Clustered Desynchronization from High-Frequency Deep Brain Stimulation. PLoS Comput Biol 2015; 11: e1004673.

3. Holt B, Wilson D, Shinn M, Moehlis J, Netoff TI. Phasic Burst Stimulation: A Closed-Loop Approach to Tuning Deep Brain Stimulation Parameters for Parkinson's Disease. PLoS Comput Biol 2016; 12: e1005011.

4. Evans JA, Elliott JA, Gorman MR. Dynamic interactions between coupled oscillators within the hamster circadian pacemaker. Behavioral Neurosci 2010; 124: 87-96.

5. Bell-Pedersen D, Cassone VM, Earnest DJ, Golden SS, Hardin PE, Thomas TL, Zoran MJ. Circadian rhythms from multiple oscillators: lessons from diverse organisms. Nat Rev Genetics 2005; 6: 544-556.

6. Chasin M. Bone Conduction Implants: The When and Why. Hearing Rev 2000.

7. Apte M, Poddar AK, Rudolph M, Rohde UL. A novel low phase noise X-band oscillator. IEEE Microwave Magazin 2015; 16: 127-135.

8. Mazzanti A, Bevilacqua A. On the phase noise performance of transformer-based CMOS differential-pair harmonic oscillators. IEEE Transact Circuits Syst I 2015; 62: 2334-2341.

9. Gouhary EL, Neihart NM. An analysis of phase noise in transformer-based dual-tank oscillators. IEEE Transact Circuits Syst I 2014; 61: 2098-2109.

10. Leeson B. A simple model of feedback oscillator noises spectrum. Proc IEEE 1966; 54: 329-330.

11. Sauvage G. Phase noise in oscillator: a mathematical analysis of Leeson model. IEEE Transact Instrument Measurement 1977; 26: 408-410.

12. Galliou S, Sthal F, Mourey M. New phase noise model for crystal oscillators application to the Clapp oscillator. IEEE Transact Ultrasonics, Ferroelectrics, Frequency Control 2003; 50: 1422-1428. 
13. Nallatamby C, Prigent $M$, Camiade M, Obregon JJ. Extension of the Leeson formula to phase noise calculation in transistor oscillators with complex tanks. IEEE Transact Microwave Theor Tech 2003; 51: 690-696.

14. Ohira T. Rigorous Q-factor formulation for one- and twoport passive linear networks from an oscillator noise spectrum viewpoint. IEEE Transact Circuits Syst II 2005; 52: $846-850$

15. Minami T, Ohira T. Unified active Q factor formula for use in noise spectrum estimation from Leeson's and Hajimiri's models. IEICE Elect Express 2013; 10: 1-6.

16. Ohira T. Extended Adler's injection locked Q factor formula for general one- and two-port active device oscillators. IEICE Elect Express 2010; 7: 1486-1492.
17. Huang XH, Tan F, Wei W, Fu W. A revisit to phase noise model of leeson. IEEE International Frequency Control Symposium Joint with the 21st European Frequency and Time Forum, 2007.

\section{*Correspondence to}

Yan Wang

School of Electrical Engineering and Information Southwest Petroleum University

PR China 